\title{
The Role of D-Dimer, Fibrinogen and C-Reactive Protein as Plasma Biomarkers in Acute Ischemic Stroke
}

\author{
Mostafa Saleh Melake ${ }^{a}$, b Rasha Ali El-Kabany ${ }^{a}$, Aktham Ismail Al-Emamª, \\ Ahmed Mohamed El-Shereef ${ }^{\mathrm{a}}$, Mohamed Okda ${ }^{\mathrm{a}}$
}

\begin{abstract}
Background: Previous studies on pathophysiology suggest a role of inflammation in atherothrombotic stroke and intracardiac thrombosis in cardioembolic stroke. We explored the magnitude of D-dimer, fibrinogen and C-reactive protein (CRP) as biomarkers in acute ischemic cerebral stroke and their relation to ischemic stroke subtypes and their impact on stroke outcome after 30 days.

Methods: The study was performed on 100 patients, admitted to Neurology Department, Menoufiya University, within 24 hours of acute ischemic stroke. Patients were subjected to clinical data collection, general and neurological examination, laboratory assessment (routine and biomarkers), brain computerized tomography (CT), magnetic resonance imaging (MRI), MRA, carotid and vertebrobasilar duplex and cardiac assessment. The patients were classified according to Trial of ORG 10172 in acute stroke treatment (TOAST) classification. Severity and disability were assessed by Scandinavian stroke scale and modified Rankin scale at admission and at 30 days.
\end{abstract}

Results: We found that D-dimer, fibrinogen and CRP were significantly higher in patients than in controls $(\mathrm{P}<0.001)$. D-dimer and fibrinogen were higher in cardioembolic stroke while CRP was higher in atherothrombotic subtype. The biomarkers were correlated significantly with the severity and disability of stroke at onset and 30 days.

Conclusions: These three biomarkers in acute ischemic stroke can be considered as non-invasive tools which add important information regarding etiology and outcome.

Keywords: D-dimer; Fibrinogen; C-reactive protein; Ischemic stroke

\section{Introduction}

Acute cerebral stroke is one of the leading causes of mortal-

Manuscript accepted for publication December 16, 2015

${ }^{a}$ Neurology Department, Menoufiya School of Medicine, Egypt ${ }^{\mathrm{b}}$ Corresponding Author: Mostafa Saleh Melake, Menoufiya School of Medicine, Neurology Department, Egypt. Email: drmostafasaleh@yahoo.com

doi: http://dx.doi.org/10.14740/jnr362w ity and the most common cause of complex chronic disability worldwide [1]. The diagnosis of stroke relies on historical data, neurologic examination, and neuroimaging techniques, including brain computerized tomography (CT) and magnetic resonance imaging (MRI) scans. A rapid blood test to confirm a clinical and imaging diagnosis of ischemic stroke based on a simple and low-cost near patient technology, would be extremely useful [2].

D-dimer and fibrinogen had been studied as biomarkers of thrombosis. Inflammation mediators such as CRP also had been investigated as markers for the diagnosis of stroke [3]. D-dimer, a marker of activation of the coagulation and fibrinolytic system has gained increasing interest over the recent years. High D-dimer levels have been reported in the acute phase of stroke [4]. Elevated D-dimer level has also been shown to relate to early clinical progression [5], stroke subtypes [6], and infarction volume [7]. Some studies have suggested that D-dimer can be seen as an outcome predictor in ischemic stroke and an indicator of severity of traumatic brain injury [8].

There is also increasing evidence that activation of the coagulation system is associated with the development and evolution of acute ischemic stroke. Fibrinogen is a thrombotic risk factor which also acts as an acute phase protein. Elevated levels of fibrinogen have been reported after stroke [4] and have been associated with increased risk of recurrent stroke [9].

Inflammation is important in ischemic stroke, both in the development of atherosclerosis and during the ischemic event [10].

Human CRP is an acute phase reactant that is rapidly unregulated by inflammatory cytokines. In line with this concept, CRP is a sensitive indicator of inflammation and has also evolved as a marker of atherosclerosis [11].

The aim of the current study was to explore the magnitude of D-dimer, fibrinogen and CRP within $24 \mathrm{~h}$ as biomarkers of acute ischemic cerebral stroke and their relation to different ischemic stroke subtypes and their impact on the short-term outcome after 30 days.

\section{Patients and Methods}

The study was performed on 100 patients aged 45 - 78 years old and 100 healthy controls matched with age and sex. The patients were 51 males and 49 females admitted to Neurology Department, Menoufiya University within $24 \mathrm{~h}$ of symptoms 
Table 1. Etiological Subtypes of the Ischemic Stroke Among Patient Group

\begin{tabular}{llc|}
\hline \multirow{2}{*}{ Etiology } & \multicolumn{2}{c}{ Patient group $(\mathbf{N}=\mathbf{1 0 0})$} \\
\cline { 2 - 3 } & No. & \% \\
\hline Atherosclerosis & 48 & 48.0 \\
Embolic & 21 & 21.0 \\
Lacunar & 17 & 17.0 \\
Undetermined & 12 & 12.0 \\
Others & 2 & 2.0
\end{tabular}

of acute cerebral stroke.

\section{Inclusion criteria}

Patients with acute cerebral infarction of either gender arriving at emergency department within $24 \mathrm{~h}$ of the symptoms onset presenting with first ever stroke were included.

\section{Exclusion criteria}

Patients with intracerebral hemorrhage, subarachnoid hemorrhage and mass lesions on brain CT or MRI, transient ischemic attack (TIA) by retrograde diagnosis, more than $24 \mathrm{~h}$ since the stroke when they arrived at the emergency department, severe hepatic or renal impairment, on anticoagulant therapy, symptoms or signs of systemic inflammatory and infectious diseases, malignancy and evidence of venous thrombotic disease on admission were excluded.

\section{Data collection}

\section{Clinical data collection}

Clinical data were collected including full history taking, socio-demographic data (age, sex, and educational level), history of vascular risk factors (hypertension defined as a systolic blood pressure $\geq 140 \mathrm{~mm} \mathrm{Hg}$ and/or a diastolic blood pressure $\geq 90 \mathrm{~mm} \mathrm{Hg}$, and/or the use of antihypertensive drugs [12]), diabetes, smoking, dyslipidemia with hypercholesterolemia and hypertriglyceridemia defined as cholesterol level $>200$ $\mathrm{mg} / \mathrm{dL}$ and triglyceride $>150 \mathrm{mg} / \mathrm{dL}$ respectively [13], cardiac diseases and current drug intake.
Table 2. Site of Infarction Among Patient Group

\begin{tabular}{lll}
\hline \multirow{2}{*}{ Site } & \multicolumn{2}{c}{ Patient group $(\mathbf{N}=\mathbf{1 0 0})$} \\
\cline { 2 - 3 } & No. & $\%$ \\
\hline Anterior cerebral artery occlusion & 8 & 8.0 \\
Middle cerebral artery occlusion & 67 & 67.0 \\
Vertebrobasilar occlusion & 20 & 20.0 \\
Multiple lacunar & 5 & 5.0 \\
\hline
\end{tabular}

Complete general and neurological examination

Complete general and neurological examination was obtained using stroke sheet.

\section{Laboratory assessment}

Blood samples were taken at admission before treatment.

1) Routine tests

Routine tests included complete blood picture, liver function tests (prothrombin time, activated partial thromboplastin time (aPTT), albumin, bilirubin and liver transaminases (SGOT and SGPT), kidney function tests (blood urea nitrogen and creatinine) random blood sugar, and erythrocyte sedimentation rate.

\section{2) Special tests}

a) Anti-double-stranded DNA, antinuclear antibody, anti-neutrophil cytoplasmic antibody-b (ANCA-b), anti-neutrophil cytoplasmic antibody-c and anticardiolipin-antibodies, and lupus anticoagulants: in suspected vasculitis disease.

b) Protein-c, protein-s, antithrombin-III and von Willebrand factor: in patients aged below 45 years, have a personal history of recurrent thrombosis without precipitating factors, thrombosis in unusual sites, thrombosis during pregnancy, and a positive family history of thrombosis.

\section{3) Plasma biomarkers}

Plasma biomarkers were D-dimer, fibrinogen and CRP within $24 \mathrm{~h}$ of acute ischemic stroke.

Table 3. Comparison Between Patient and Control Groups Regarding D-Dimer, Fibrinogen and CRP

\begin{tabular}{lllll} 
Parameters & Patient group $(\mathbf{N}=\mathbf{1 0 0})($ mean $\pm \mathbf{S D})$ & Control group $(\mathbf{N}=100)($ mean \pm SD) & Mann- Whitney test & P value \\
\hline D-dimer $(\mathrm{ng} / \mathrm{mL})$ & $635.5 \pm 699.6$ & $99.1 \pm 53.8$ & 8.59 & $<0.001^{* *}$ \\
Fibrinogen $(\mathrm{mg} / \mathrm{dL})$ & $365.4 \pm 151.8$ & $211.9 \pm 15.7$ & 8.66 & $<0.001^{* *}$ \\
CRP $(\mathrm{mg} / \mathrm{L})$ & $5.2 \pm 3.9$ & $0.90 \pm 0.33$ & 10.76 & $<0.001^{* *}$ \\
\hline
\end{tabular}

**Highly significant at $\mathrm{P}<0.001$. 
Table 4. Comparison Between the Subtypes of Stroke (According to Etiology) Regarding D-Dimer, Fibrinogen and CRP

\begin{tabular}{|c|c|c|c|c|c|c|c|}
\hline & $\begin{array}{l}\text { Atherothrombotic } \\
(\mathrm{N}=48)\end{array}$ & $\begin{array}{l}\text { Cardioembolic } \\
(\mathrm{N}=21)\end{array}$ & $\begin{array}{l}\text { Lacunar } \\
(\mathrm{N}=17)\end{array}$ & $\begin{array}{l}\text { Undetermined/ } \\
\text { others }(\mathrm{N}=14)\end{array}$ & Test & P value & Post hoc test \\
\hline D-dimer & $513.4 \pm 574.3$ & $1259.2 \pm 954.5$ & $317.1 \pm 238.1$ & $500.3 \pm 407.7$ & $\begin{array}{l}\text { Kruskal-Wallis } \\
\text { test }=15.41\end{array}$ & $0.001 *$ & $\begin{array}{l}\mathrm{P} 1=0.02 * \\
\mathrm{P} 2=0.26 \\
\mathrm{P} 3=1.00 \\
\mathrm{P} 4=0.001 * \\
\mathrm{P} 5=0.03 * \\
\mathrm{P} 6=0.81\end{array}$ \\
\hline
\end{tabular}

*Significant. **Highly significant.

\section{Radiological assessment}

a) Brain CT was done at admission, focusing on the early infarction signs (focal brain swelling, early hypodensity, attenuation of basal ganglia, or hyperdense artery sign) and excluding cerebral hemorrhage or any focal lesion. b) Brain MRI to determine the territory and the size of infarction (small $\leq 1.5$ $\mathrm{cm}$, large $>1.5 \mathrm{~cm}$ according to TOAST classification) and MRA (MR angiography) within $24-72 \mathrm{~h}$ of admission were done.

\section{Neurosonological assessment}

Extracranial vessels ultrasound examination: common carotid artery (CCA), internal carotid (ICA) and extracranial vertebral arteries were visualized by combined B-mode and colorDoppler ultrasound on both sides combined with pulsed wave Doppler.

\section{Cardiac evaluation}

Cardiac evaluation included ECG, transthoracic and transesophageal echocardiography.

All patients were classified according to TOAST classification into atherothrombotic, cardioembolic, lacunar, undetermined etiology and stroke of other etiology.

\section{Assessment of severity and disability}

For assessment of stroke severity and disability, Scandinavian stroke scale [14] and modified Rankin scale were done at the time of admission and at 30 days after the onset.

\section{Results}

In this study, it was found that there were no significant differences between patients and controls regarding age and sex but there was a significant difference as regards hypertension, diabetes mellitus, smoking, ischemic heart disease and atrial fibrillation. There was a significant difference between the two groups regarding all items of the lipid profile (cholesterol, triglycerides, HDL and LDL).

The etiology of the ischemic stroke among the 100 patients was atherothrombotic in 48 patients $(48.0 \%)$, embolic in 21 patients $(21.0 \%)$, lacunar in 17 patients $(17.0 \%)$, undetermined in 12 patients $(12.0 \%)$ and other etiology in two patients $(2.0 \%)$ (Table 1$)$. The site of the cerebral infarction was in anterior cerebral artery territory in eight patients $(8 \%)$, middle cerebral artery territory in 67 patients $(67 \%)$, vertebrobasilar artery territory in 20 patients $(20 \%)$ and multiple lacunar in five patients (5\%) (Table 2).

D-dimer, fibrinogen and CRP were highly significantly different $(\mathrm{P}<0.001)$ between the patients and controls (Table 3).

There was highly significant difference between the subtypes regarding $\mathrm{D}$-dimer $(\mathrm{P}=0.001)$ with the highest value in the cardioembolic group and lowest in the lacunar group. There was a significant difference between the cardioembolic and atherosclerotic groups $(\mathrm{P}=0.02)$ and between the cardioembolic and lacunar groups $(\mathrm{P}=0.001)$ and between cardioembolic and undermined/other groups $(\mathrm{P}=0.03)$. There was a significant difference between the different subtypes of stroke 
Table 5. Spearman Correlation Between D-Dimer, Fibrinogen, CRP and Scandinavian Stroke Scale and Modified Rankin Scale at Admission

\begin{tabular}{lllllllll} 
& \multicolumn{2}{c}{ D-dimer } & & \multicolumn{2}{c}{ Fibrinogen } & & \multicolumn{2}{c}{ CRP } \\
\cline { 2 - 3 } & $\mathbf{R}$ & P-value & & $\mathbf{R}$ & P-value & & r & P-value \\
\hline SSS & -0.464 & $<0.001^{* *}$ & & -0.492 & $<0.001^{* *}$ & & -0.367 & $<0.001^{* *}$ \\
mRs & 0.397 & $<0.001^{* *}$ & & 0.357 & $<0.001^{* *}$ & & 0.207 & $0.04 *$ \\
\hline
\end{tabular}

*Significant. **Highly significant.

regarding fibrinogen $(\mathrm{P}=0.004)$ with the highest value in the cardioembolic group and lowest in the lacunar group. There was a significant difference between atherosclerotic and lacunar groups $(\mathrm{P}=0.002)$ and between cardioembolic and lacunar groups $(\mathrm{P}=0.001)$. There was highly significant difference between the different subtypes $(\mathrm{P}<0.001)$ with the highest value in the atherosclerotic group and lowest in the lacunar group.

There was highly significant differences between atherosclerotic and lacunar groups $(\mathrm{P}<0.001)$, highly significant differences between embolic and lacunar groups $(\mathrm{P}<0.001)$ and significant difference between lacunar and undermined/other groups and significant difference between atherosclerotic and undetermined/other groups (Table 4).

As regards relation between the biomarkers and initial severity and disability on admission, there was highly significant inverse correlation between D-dimer, fibrinogen and CRP and initial Scandinavian stroke scale $(\mathrm{P}<0.001)$. There was highly significant correlation between $\mathrm{D}$-dimer, fibrinogen $(\mathrm{P}$ $<0.001)$ and initial modified Rankin scale. Also, there was significant correlation between CRP and initial modified Rankin scale (Table 5).

At 30 days after the onset, there was highly significant inverse correlation between D-dimer, fibrinogen and CRP at admission and the severity of stroke after 30 days assessed by Scandinavian stroke scale $(\mathrm{P}<0.001)$ and a significant correlation between D-dimer, fibrinogen and CRP at admission and the disability of stroke after 30 days assessed by modified Rankin scale (Table 6).

There was a significant correlation between D-dimer, fibrinogen and CRP done at admission and size of the infarction.

\section{Discussion}

The results of this study showed that D-dimer was significantly higher in patients with acute ischemic stroke when compared with control subjects. This agreed with Lip et al [15] who found that baseline D-dimer levels measured within $<12 \mathrm{~h}$ from the onset of acute stroke were significantly higher than those in healthy controls. Also Ageno et al [16] found that Ddimer levels on day 1 of the acute ischemic stroke were significantly higher in patients than those of controls.

The result of this study was in contrast to Haapaniemi and Tatlisumak [17] who postulated that D-dimer assessment cannot be used as an acute ischemic stroke index, with the exception of the cardioembolic subtype.

The results of the present study showed that there was highly significant difference between the patients and control group as regards fibrinogen. This coincides with Shenhar-Tsarfaty et al [18] who found that fibrinogen concentrations were significantly elevated in patients with acute ischemic stroke within $24 \mathrm{~h}$ when compared with matched controls.

There was highly significant difference between the patients and controls regarding CRP which agreed with Hasan et al [19] who found that CRP within $24 \mathrm{~h}$ of the onset of the symptoms was significantly differentiated between ischemic stroke and healthy control subjects.

The result of the present study showed that D-dimer was highest in the cardioembolic group and lowest in the lacunar group. There was a significant difference between cardioembolic and atherosclerotic groups and between cardioembolic and lacunar groups and between cardioembolic and undermined/other groups. This coincides with Liu [20] who found that D-dimer levels were significantly different among the subtypes and were the highest in cardioembolic followed by large artery atherosclerosis and small artery occlusion. In contrast to our results, Takano et al [21] found marked elevation of plasma concentration of D-dimer was observed only in cardioembolic versus control within $48 \mathrm{~h}$ of onset persisting for 1 month with a gradual decline. In atherothrombotic stroke, the level of D-dimer was not elevated at the onset, but increased 7 days after onset and no significant changes in these marker levels were observed in lacunar stroke. The result of the present study showed that there was significant difference between the different subtypes of stroke regarding fibrinogen $(\mathrm{P}=0.004)$ with the highest value in the cardioembolic group

Table 6. Spearman Correlation Between D-Dimer, Fibrinogen, CRP and the Outcome of the Stroke Using Scandinavian Stroke Scale and Modified Rankin Scale at 30 Days

\begin{tabular}{|c|c|c|c|c|c|c|}
\hline & \multicolumn{2}{|c|}{ D-dimer } & \multicolumn{2}{|c|}{ Fibrinogen } & \multicolumn{2}{|r|}{ CRP } \\
\hline & $\mathbf{R}$ & P-value & $\mathbf{R}$ & P-value & $\mathbf{r}$ & P-value \\
\hline SSS & -0.496 & $<0.001 * *$ & -0.473 & $<0.001 * *$ & -0.386 & $<0.001 * *$ \\
\hline $\mathrm{mRs}$ & 0.326 & $0.001 *$ & 0.336 & $0.001 *$ & 0.227 & $0.03 *$ \\
\hline
\end{tabular}

*Significant. **Highly significant. 
and lowest in the lacunar group. There were significant differences between atherosclerotic and lacunar groups and between cardioembolic and lacunar groups. This was in agreement with Alvarez-Perez et al [22] who found that the highest median level of fibrinogen was found in patients with cardioembolic stroke followed by patients with atherothrombotic stroke, but differences among subtypes were not significant. Turaj et al [23] showed that the median plasma concentration of fibrinogen was higher in patients with large vessel disease than in patients with small vessel disease $(\mathrm{P}=0.0001)$. In contrast to our study, Lang et al [24] found that fibrinogen was highest in large artery atherosclerosis but the distribution between the different subtypes of acute ischemic stroke was statistically insignificant. In the present study, there was highly significant difference between the different subtypes as regard to CRP with the highest value in the atherosclerotic group and lowest in the lacunar group. There were highly significant differences between atherosclerotic and lacunar groups, highly significant differences between cardioembolic and lacunar groups and significant difference between lacunar and undermined/other groups. This is in agreement with Alvarez-Perez et al [22] who found that the median level of CRP was higher in patients with atherothrombotic stroke, followed by cardioembolic stroke. Differences were significant when patients with atherothrombotic stroke were compared with those with lacunar and undetermined strokes.

The result of the present study showed a significant correlation between the D-dimer levels and the size of the cerebral infarction which agreed with Yang et al [25] who found that there was a positive correlation between levels of D-dimer and the infarct volume. For fibrinogen and CRP, the results of the present study showed a significant correlation between both biomarkers and size of the infarction which coincided with agreement with Yigun and Bakirci [26] who found that patients with large infarcts in the median cerebral artery and anterior cerebral artery had higher fibrinogen and CRP concentrations than the control group. However, no difference was seen in their levels between the patients with an infarct in the posterior cerebral artery and controls.

In the present study, D-dimer, fibrinogen and CRP had a significant relation with stroke severity and disability on admission which agreed with $\mathrm{Zi}$ and Shuai [27] who found that plasma D-dimer levels on admission in patients with acute ischemic stroke increased with increasing severity of stroke as defined by the NIHSS score. Also, it agreed with Ufuk et al [28] who found significant relationship between fibrinogen levels of patients and stroke severity score.

The present study showed that D-dimer, fibrinogen and CRP had significant relation with poor outcome at 30 days which agreed with Yang et al [25] who found that elevated levels of D-dimer were short-term prognostic marker of outcome and death in patients with acute ischemic stroke, also it agreed with De Zoppo et al [29] who found that the proportion of patients with good functional outcome decreased with increasing initial fibrinogen levels and patients with initial fibrinogen levels $<450 \mathrm{mg} / \mathrm{dL}$ had better outcomes. In agreement with our study, Hamidon et al [30] found that elevated CRP level in acute ischemic stroke was a predictor of severe functional disability at 1 month and was also associated with larger infarcts.

\section{Conclusion}

Our biomarker panel takes its strength from its coverage of the underlying pathophysiology of ischemic stroke. In addition, we found that information provided by this biomarker panel very useful in detecting the etiology in these patients with acute cerebrovascular stroke. These information may provide a rational basis by which to choose the optimal treatment and may help stratify patients in clinical trials of stroke treatment.

\section{Conflict of Interest}

All authors declare no conflict of interest.

\section{References}

1. Flynn RW, MacWalter RS, Doney AS. The cost of cerebral ischaemia. Neuropharmacology. 2008;55(3):250256.

2. Whiteley W, Tseng MC, Sandercock P. Blood biomarkers in the diagnosis of ischemic stroke: a systematic review. Stroke. 2008;39(10):2902-2909.

3. Allard L, Burkhard PR, Lescuyer P, Burgess JA, Walter N, Hochstrasser DF, Sanchez JC. PARK7 and nucleoside diphosphate kinase A as plasma markers for the early diagnosis of stroke. Clin Chem. 2005;51(11):2043-2051.

4. Reganon E, Vila V, Martinez-Sales V, Vaya A, Lago A, Alonso P, Aznar J. Association between inflammation and hemostatic markers in atherothrombotic stroke. Thromb Res. 2003;112(4):217-221.

5. Yuan W, Shi ZH. The relationship between plasma D-dimer levels and outcome of Chinese acute ischemic stroke patients in different stroke subtypes. J Neural Transm (Vienna). 2014;121(4):409-413.

6. Koch HJ, Horn M, Bogdahn U, Ickenstein GW. The relationship between plasma D-dimer concentrations and acute ischemic stroke subtypes. J Stroke Cerebrovasc Dis. 2005;14(2):75-79.

7. Park YW, Koh EJ, Choi HY. Correlation between Serum D-Dimer Level and Volume in Acute Ischemic Stroke. J Korean Neurosurg Soc. 2011;50(2):89-94.

8. Hudak AM, Caesar RR, Frol AB, Krueger K, Harper CR, Temkin NR, Dikmen SS, et al. Functional outcome scales in traumatic brain injury: a comparison of the Glasgow Outcome Scale (Extended) and the Functional Status Examination. J Neurotrauma. 2005;22(11):1319-1326.

9. Woodward M, Lowe GD, Campbell DJ, Colman S, Rumley A, Chalmers J, Neal BC, et al. Associations of inflammatory and hemostatic variables with the risk of recurrent stroke. Stroke. 2005;36(10):2143-2147.

10. Chamorro A. Role of inflammation in stroke and atherothrombosis. Cerebrovasc Dis. 2004;17(Suppl 3):1-5.

11. Bassuk SS, Rifai N, Ridker PM. High-sensitivity C-reactive protein: clinical importance. Curr Probl Cardiol. 2004;29(8):439-493.

12. Chobanian AV, Bakris GL, Black HR, Cushman WC, 
Green LA, Izzo JL, Jr., Jones DW, et al. Seventh report of the Joint National Committee on Prevention, Detection, Evaluation, and Treatment of High Blood Pressure. Hypertension. 2003;42(6):1206-1252.

13. Miller M, Stone NJ, Ballantyne C, Bittner V, Criqui MH, Ginsberg HN, Goldberg AC, et al. Triglycerides and cardiovascular disease: a scientific statement from the American Heart Association. Circulation. 2011;123(20):22922333.

14. Multicenter trial of hemodilution in ischemic strokebackground and study protocol. Scandinavian Stroke Study Group, 1985.

15. Lip GY, Blann AD, Farooqi IS, Zarifis J, Sagar G, Beevers DG. Sequential alterations in haemorheology, endothelial dysfunction, platelet activation and thrombogenesis in relation to prognosis following acute stroke: The West Birmingham Stroke Project. Blood Coagul Fibrinolysis. 2002;13(4):339-347.

16. Ageno W, Finazzi S, Steidl L, Biotti MG, Mera V, Melzi D'Eril G, Venco A. Plasma measurement of D-dimer levels for the early diagnosis of ischemic stroke subtypes. Arch Intern Med. 2002;162(22):2589-2593.

17. Haapaniemi E, Tatlisumak T. Is D-dimer helpful in evaluating stroke patients? A systematic review. Acta Neurol Scand. 2009;119(3):141-150.

18. Shenhar-Tsarfaty S, Ben Assayag E, Bova I, Shopin L, Cohen M, Berliner S, Shapira I, et al. Persistent hyperfibrinogenemia in acute ischemic stroke / transient ischemic attack (TIA). Thromb Haemost. 2008;99(1):169-173.

19. Hasan N, McColgan P, Bentley P, Edwards RJ, Sharma P. Towards the identification of blood biomarkers for acute stroke in humans: a comprehensive systematic review. $\mathrm{Br}$ J Clin Pharmacol. 2012;74(2):230-240.

20. Liu LB, Li M, Zhuo WY, Zhang YS, Xu AD. The role of hs-CRP, D-dimer and fibrinogen in differentiating etiological subtypes of ischemic stroke. PLoS One. 2015;10(2):e0118301.

21. Takano K, Yamaguchi T, Uchida K. Markers of a hyper- coagulable state following acute ischemic stroke. Stroke. 1992;23(2):194-198.

22. Alvarez-Perez FJ, Castelo-Branco M, Alvarez-Sabin J. Usefulness of measurement of fibrinogen, D-dimer, Ddimer/fibrinogen ratio, $\mathrm{C}$ reactive protein and erythrocyte sedimentation rate to assess the pathophysiology and mechanism of ischaemic stroke. J Neurol Neurosurg Psychiatry. 2011;82(9):986-992.

23. Turaj W, Slowik A, Pulyk R, Adamski M, Szczudlik A. [Comparison of plasma concentrations of fibrinogen in patients with ischemic stroke due to large vessel disease and small vessel disease]. Neurol Neurochir Pol. 2006;40(4):297-301.

24. Lang Q, Zhou M, Feng H, Guo J, Chen N, He L. Research on the relationship between fibrinogen level and subtypes of the TOAST criteria in the acute ischemic stroke. BMC Neurol. 2013;13(207.

25. Yang XY, Gao S, Ding J, Chen Y, Zhou XS, Wang JE. Plasma D-dimer predicts short-term poor outcome after acute ischemic stroke. PLoS One. 2014;9(2):e89756.

26. Iyigun I, Bakirci Y. Plasma concentrations of C-reactive protein and fibrinogen in ischaemic stroke. J Int Med Res. 2002;30(6):591-596.

27. Zi WJ, Shuai J. Plasma D-dimer levels are associated with stroke subtypes and infarction volume in patients with acute ischemic stroke. PLoS One. 2014;9(1):e86465.

28. Ufuk E, Ufuk E, Aysun U, Ozlem C, H.Tugrul A, Hulya Y, Umit G, E.Levent I. The Role Of Acute Phase Reactants In Acute Ischemic Stroke. Journal of Neurological Sciences (Turkish). 2007;24(1):64-69.

29. del Zoppo GJ, Levy DE, Wasiewski WW, Pancioli AM, Demchuk AM, Trammel J, Demaerschalk BM, et al. Hyperfibrinogenemia and functional outcome from acute ischemic stroke. Stroke. 2009;40(5):1687-1691.

30. Hamidon BB, Sapiah S, Nawawi H, Raymond AA. The prognostic value of C-reactive protein (CRP) levels in patients with acute ischaemic stroke. Med J Malaysia. 2004;59(5):631-637. 